\title{
Recurrence of permanent junctional re-entry tachycardia: indication for ablation of the junctional pathway
}

Submitted: 26 April 2016; Accepted: 16 June 2016; Published online: 21 June 2016

Four months ago we treated a 43-year old male patient with a tachycardia-induced cardiomyopathy due to permanent junctional re- entry tachycardia who presented with severe dilatation and reduction of left ventricular function. After administration of bisoprolol, the patient resumed sinus rhythm thus ablation of the junctional pathway was not considered necessary [1]. Subsequently, the patient stopped wearing a life vest and stopped taking his prescribed bisoprolol and an ACE inhibitor because of very poor compliance

The patient was readmitted to hospital because of edema and dyspnoea.

ECG revealed again permanent junctional re-entry tachycardia with negative T-waves in inferior leads (Figure 1).

Echocardiography showed a severe dilatation of the left ventricle of $6.8 \mathrm{~cm}$ and a severe reduction of left ventricular function with an ejection fraction of $28 \%$. Furthermore, moderate mitral and tricuspid regurgitation was documented.

After two days resuming of the prior dosage of bisoprolol, the patient still was in incessant permanent junctional re-entry tachycardia,
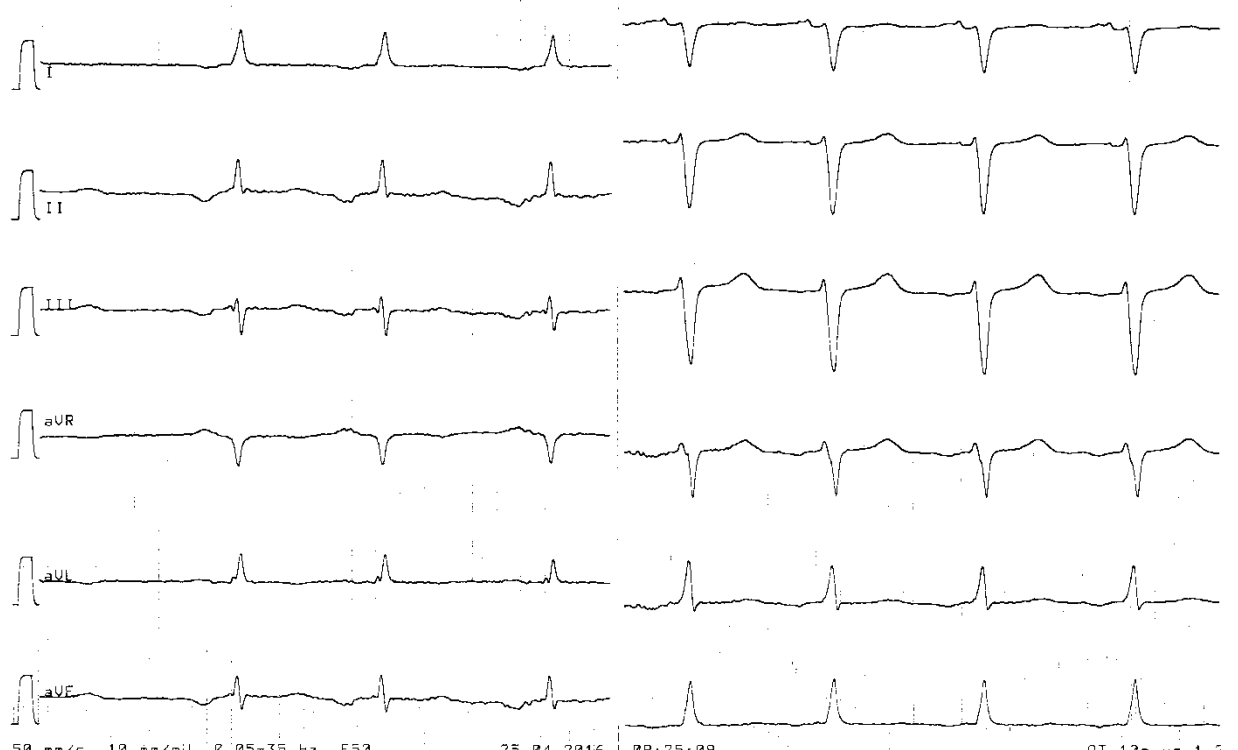

$50 \mathrm{~mm} / \mathrm{s}$ id $\mathrm{mm} / \mathrm{mil} \mathrm{z}$ Q Q

$25.84 .2016 \quad 08: 25: 89$
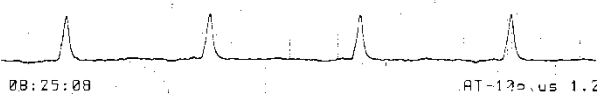

Figure 1: ECG of the patient with permanent junctional re-entry tachycardia by negative $\mathrm{P}$ waves in lead II, III, aVF. 
which lead to dramatic deterioration of left ventricular function. This patient was send home and was booked for radiofrequency ablation in an electrophysiological laboratory not far away.

PJRT is a potentially lethal arrhythmia in children and in adolescants with tachycardia induced cardiomyopathy. Although rarely reported, spontaneous resolution is not uncommon. Antiarrhythmic treatment is often effective, especially with amiodarone and verapamil. Radiofrequency ablation should be reserved for older patients and especially in patients with persistent left ventricular dysfunction.

Radiofrequency ablation of this particular accessory pathway remains an intervention at high risk of requiring permanent pacemaker implantation because of its proximity to the septum. The primary ablation success rate is $92 \%$, but the recurrence rate

\section{References}

1. Peters S. Tachycardiomyopathy: A case of dilated cardiomyopathy due to permanent junctional reentrant tachycardia. Int. J. Cardiol. 207, 233-234 (2016).

2. Nadji G, Hermida JS, Kubala M, et al. Cryoablation of junctional tachycardia at high risk of atrio-ventricular block. Arch. Cardiovasc. Dis. 101, 149-154 (2008). after 9 months is $29 \%$ for cryoablation and $8.6 \%$ for radiofrequency ablation [2].

In nearly all patients left ventricular function recovers when PJRT is stopped [3]. Despite normalization of left ventricular function, however, a few patients die likely due to structural abnormalities. Experimental data have shown that radiofrequency lesions appear to increase in size with somatic growth, with replacement of normal myocytes with fibrous and elastic tissue forming a potential arrhythmogenic substrate [4].

In adults tachycardia-induced cardiomyopathy is accomplanied by slower tachycardia rates than in children [3]. Radiofrequency catheter ablation is highly effective and should be considered as treatment of first choice in adult patients.

3. Meiltz A, Weber R, Halimi F, et al. Permanent form of junctional reciprocating tachycardia in adults: peculiar features and results of radiofrequency catheter ablation. Europace. 8, 21-28 (2006).

4. Vaksmann G1, D'Hoinne C, Lucet, et al. Permanent junctional reciprocating tachycardia in children: a multicentre study on clinical profile and outcome. Heart. 92, 101-104 (2006). 